\title{
ON THE TWO DEFINITIONS OF THE CONLEY INDEX
}

\author{
HENRY L. KURLAND
}

(Communicated by Kenneth R. Meyer)

\begin{abstract}
The two definitions of the homotopy equivalences between Conley index spaces of an isolated invariant set, the original one of Conley [C] as completed by the author in [K1] and the more recent definition of Salamon [S], are shown to define the same homotopy classes without reference to the difficult proof of [K1] showing the Conley index to be a connected simple system. The equivalences of the original definition are useful in describing certain geometric situations in terms of the index; examples are given.
\end{abstract}

\section{INTRODUCTION}

Let $X$ be a locally compact Hausdorff space, assume $X \times\{0\} \subset G \subset X \times \mathbf{R}$ with $G$ open in $X \times \mathbf{R}$, and for $(x, t) \in G$, let $(x, t) \mapsto x \cdot t$ denote a continuous local flow on $X$. Further, for $x \in X$ assume the map $t \mapsto x \cdot t$, defined for $t$ such that $(x, t) \in G$, has closed, connected graph in $\mathbf{R} \times X$. Call an orbit of this flow through a point $x \in X$ complete whenever $\{x\} \times \mathbf{R} \subset G$. Then $S \subset X$ is invariant if it is the union of complete orbits of the flow, the empty union not excluded. Note that the arbitrary union of invariant sets is invariant as is the topological closure of a relatively compact invariant set. (The relative compactness is not needed if $G=X \times \mathbf{R}$.) Therefore any compact subset of $X$ contains a largest, but possibly empty, invariant subset which itself is compact. A compact set $N \subset X$ is an isolating neighborhood of the flow if the largest invariant subset of $N$ lies in the interior of $N$, and an invariant set $S$ is isolated if it is the largest invariant subset of some isolating neighborhood.

Conley in [C] defined an index for each isolated invariant set $S$, denoted here by $\mathscr{I}(S)$, that is useful in proving existence theorems for differential equations. This index takes the form of a small subcategory of the homotopy category of topological spaces with base point whose objects, called index spaces, are formed as quotient spaces from certain distinguished pairs of subsets of $X$, called index pairs (see Figures 1 and 2 for examples), and whose morphisms are the

Received by the editors January 20, 1989.

1980 Mathematics Subject Classification (1985 Revision). Primary 58F25, 58F35, 34C35; Secondary 34B15, 34E 15 .

Key words and phrases. Isolated invariant set, Conley index, connected simple system, homology of the Conley index, boundary layer, common squeeze time, flow map. 
homotopy classes of certain flow and inclusion induced homotopy equivalences between the index spaces there being exactly one morphism between any two index spaces, which by definition makes $\mathscr{I}(S)$ a connected simple system.

There are two definitions of these morphisms: the original one given by Conley in [C] and completed by the author in [K1], and the more recent and elegantly simple definition given by Salamon in [S]. In this note, it will be shown that the two definitions yield the same small category. This is accomplished by showing that each inclusion and flow induced homotopy equivalence of the original construction is homotopic to a flow induced homotopy equivalence defined in [S], and conversely, that each flow induced homotopy equivalence of [S] has a factorization in terms of inclusion and flow induced homotopy equivalences defined in $[\mathrm{C}]$ and $[\mathrm{K} 1]$. Further, this is done without reference to the complicated proof of [K1] that $\mathscr{I}(S)$ is a connected simple system. Thus, the inclusion and flow-induced maps used to define the morphisms in the original construction can be regarded as defining morphisms in the Conley index as defined in [S]. This is quite useful in describing certain geometric situations in terms of the index since the maps of the original construction are more easily interpreted geometrically. One such situation, examined briefly at the end of the paper, involves finding a nested family of geometric representatives of a non-zero homology class in the index such that the intersection of the representatives lies in an invariant manifold of the isolated invariant set and arises in [K4] and [K5] with regard to determining the asymptotoic behavior of solutions to certain singularly perturbed two-point boundary value problems on a finite interval where the geometric representatives lie in the locus of points satisfying a boundary condition at one of the endpoints. Another, which for reasons of length will not be discussed here, arises in the definition of the splitting map of a repellerattractor pair $[\mathrm{K} 2, \S 4]$ which can be used to detect or reflect the presence of a heteroclinic orbit between isolated invariant sets [K3, Theorem 3.3] and, consequently, is used in [K5] to detect internal transition layers in solutions to the aforementioned two-point boundary value problems.

For the rest of this note let $S$ be a fixed isolated invariant set of the local flow on $X$. Recall that if $N_{1}$ and $N_{0}$ are compact subsets of $X$, then $\left\langle N_{1}, N_{0}\right\rangle$ is an index pair for $S$ if, and only if,

(i) $\operatorname{cl}\left(N_{1} \backslash N_{0}\right)$ is an isolating neighborhood of $S$;

(ii) $x \in N_{0}, t>0$, and $x \cdot[0, t] \subset N_{1}$ implies $x \cdot[0, t] \subset N_{0}$;

(iii) $x \in N_{1}$ and $x \cdot \mathbf{R}^{+} \not \subset N_{1}$ implies there exists $t \geq 0$ so that $x \cdot[0, t] \subset N_{1}$ and $x \cdot t \in N_{0}$.

Properties (i), (ii), and (iii) are, respectively, the isolating, relative positive invariance, and exit properties of an index pair. Existence of index pairs is shown in [C] and [S]. The index space associated to an index pair $\left\langle N_{1}, N_{0}\right\rangle$ is the quotient space $N_{1} / N_{0}$, i.e., when $N_{1} \cap N_{0}$ is nonempty, $N_{1} / N_{0}$ is the pointed space obtained from $N_{1}$ by collapsing $N_{1} \cap N_{0}$ to a point, denoted [ $\left.N_{0}\right]$, and taking $\left[N_{0}\right]$ as basepoint, and when $N_{1} \cap N_{0}$ is empty, with $\{*\}$ any 
one point space disjoint from $N_{1}, N_{1} / N_{0}$ is the disjoint union $N_{1} \cup\{*\}$ with basepoint $*$ which for convenience will also be denoted $\left[N_{0}\right]$. In both cases $[x]$ denotes the image of a point $x \in N_{1}$ under the quotient map $N_{1} \rightarrow N_{1} / N_{0}$.

\section{SALAmon's DEFINITION of $\mathscr{I}(S)$}

Let $\left\langle N_{1}, N_{0}\right\rangle$ and $\left\langle\tilde{N}_{1}, \tilde{N}_{0}\right\rangle$ be index pairs for $S$. With the additional assumption that $X$ is metric (this assumption is unnecessary as pointed out in $\S 3$ below), Salamon shows that there exists $T \geq 0$ so that $t \geq T$ implies

(1) $x \cdot[-t, t] \subset N_{1} \backslash N_{0}$ implies $x \in \tilde{N}_{1} \backslash \tilde{N}_{0}$;

(2) $x \cdot[-t, t] \subset \tilde{N}_{1} \backslash \tilde{N}_{0}$ implies $x \in N_{1} \backslash N_{0}$.

For reasons described later, $T$ will be referred to as a common squeeze time for the index pairs $\left\langle N_{1}, N_{0}\right\rangle$ and $\left\langle\tilde{N}_{1}, \tilde{N}_{0}\right\rangle$.

Given a common squeeze time $T$ for the index pairs $\left\langle N_{1}, N_{0}\right\rangle$ and $\left\langle\tilde{N}_{1}, \tilde{N}_{0}\right\rangle$ and $t \geq T$, Salamon defines $F^{t}: N_{1} / N_{0} \rightarrow \bar{N}_{1} / \bar{N}_{0}$ by the formula

$F^{t}([x]):=F([x], t):= \begin{cases}{[x \cdot 3 t]} & \text { if } x \cdot[0,2 t] \subset N_{1} \backslash N_{0}, x \cdot[t, 3 t] \subset \tilde{N}_{1} \backslash \tilde{N}_{0}, \\ {\left[\tilde{N}_{0}\right]} & \text { otherwise }\end{cases}$

and shows that $F: N_{1} / N_{0} \times\left[T, \infty\left[\rightarrow \tilde{N}_{1} / \tilde{N}_{0}\right.\right.$ is continuous. Let us call each $F^{t}, t \geq T$, a flow map from the index space $N_{1} / N_{0}$ to the index space $\tilde{N}_{1} / \tilde{N}_{0}$.

Salamon defines the homotopy class of each flow map between index spaces to be a morphism in the Conley index. Since the path of maps $F^{(1-r) s+r t}$, $0 \leq r \leq 1$, describes a homotopy from $F^{s}$ to $F^{t}$ whenever $s, t \geq T$, the flow maps from one index space to another have a common homotopy class; i.e., essentially by definition in [S], there is exactly one morphism in $\mathscr{I}(S)$ from one index space for $S$ to another. Amazingly little work must then be done to show that each flow map is a homotopy equivalence and that $\mathscr{I}(S)$ is a category under the usual composition of homotopy classes; i.e., the collection of homotopy classes of flow maps is closed under composition and when $N_{i}=\tilde{N}_{i}$, $i=0,1$, the homotopy class of $F^{t}$ is that of the identity on $N_{1} / N_{0}$.

Specifically, if $\left\langle M_{1}, M_{0}\right\rangle,\left\langle N_{1}, N_{0}\right\rangle$, and $\left\langle P_{1}, P_{0}\right\rangle$ are index pairs for $S, T \geq$ 0 is chosen large enough so that it is a common squeeze time for each of the three pairs of index pairs taken from these three, and $F_{1}^{t}: M_{1} / M_{0} \rightarrow N_{1} / N_{0}$, $F_{2}^{t}: N_{1} / N_{0} \rightarrow P_{1} / P_{0}$, and $F_{3}^{t}: M_{1} / M_{0} \rightarrow P_{1} / P_{0}$ denote the flow maps between the index spaces for $t \geq T$, then it is a straightforward exercise following from the definition of "common squeeze time" to show that $F_{2}^{t} \circ F_{1}^{t}=F_{3}^{2 t}$; hence the composition of the homotopy classes of two flow maps is the homotopy class of a flow map. In particular, if $M_{i}=P_{i}, i=0,1$, it is immediate that $\left\langle M_{1}, M_{0}\right\rangle$ and $\left\langle P_{1}, P_{0}\right\rangle$ have common squeeze time zero and that $F_{3}^{0}=1_{M_{1} / M_{0}}$; hence the homotopy class of $F_{3}^{t}, t \geq 0$, is that of $1_{M_{1} / M_{0}}$. It follows immediately that 
each flow map between index spaces has a flow map as homotopy inverse and is thereby a homotopy equivalence.

\section{Conley'S DEFINITION OF $\mathscr{I}(S)$}

The original definition of $\mathscr{I}(S)$ as outlined in [C] requires the construction of auxiliary index pairs from a given index pair. Given any index pair $\left\langle N_{1}, N_{0}\right\rangle$ for $S$ and $t \geq 0$ define

$$
\begin{aligned}
N_{1}^{t} & :=\left\{x \in N_{1}: x \cdot[-t, 0] \subset N_{1}\right\}, \\
N_{0}^{-t} & :=\left\{x \in X: \exists s, 0 \leq s \leq t \text { and } x \cdot s \in N_{0}\right\} .
\end{aligned}
$$

It is shown in [K1] that for $s, t \geq 0,\left\langle N_{1}, N_{0}^{-t}\right\rangle,\left\langle N_{1}^{t}, N_{0}\right\rangle$ and $\left\langle N_{1}^{s}, N_{0}^{-t}\right\rangle$ are also index pairs for $S$.

The notion of a common squeeze time for index pairs is easily recast in terms of these auxiliary index pairs as follows. Note that $x \in N_{1}^{t} \backslash N_{0}^{-t}$ if, and only if, $x \cdot[-t, t] \subset N_{1} \backslash N_{0}$ with the analogous remark holding when $\tilde{N}_{i}$ replaces $N_{i}$, $i=0,1$. Thus $T \geq 0$ is a common squeeze time for the index pairs $\left\langle N_{1}, N_{0}\right\rangle$ and $\left\langle\tilde{N}_{1}, \tilde{N}_{0}\right\rangle$ for $S$ if, and only if, $t \geq T$ implies

(1') $N_{1}^{t} \backslash N_{0}^{-t} \subset \tilde{N}_{1} \backslash \tilde{N}_{0}$;

(2') $\tilde{N}_{1}^{t} \backslash \tilde{N}_{0}^{-t} \subset N_{1} \backslash N_{0}$.

The proof of existence and continuity of flow maps in [S] uses the metricity of $X$ only in finding a common squeeze time; hence the definition of $\mathscr{I}(S)$ of $\S 2$ is valid when $X$ is only locally compact and Hausdorff as a consequence of the following discussion. Lemma 3.3(3) of [K1], which does not require $X$ metric, states that given a neighborhood of $S$ and an index pair $\left\langle N_{1}, N_{0}\right\rangle$, there exists $T \geq 0$ so that $N_{1}^{t} \backslash N_{0}^{-t}$ lies in the interior of the neighborhood whenever $t \geq T$. As $\tilde{N}_{1} \backslash \tilde{N}_{0}$ is a neighborhood of $S$, applying the lemma yields $T_{1}$ so that $t \geq T_{1}$ implies $\left(1^{\prime}\right)$ holds, and interchanging the roles of $\left\langle N_{1}, N_{0}\right\rangle$ and $\left\langle\tilde{N}_{1}, \tilde{N}_{0}\right\rangle$ yields $T_{2} \geq 0$ so that $t \geq T_{2}$ implies $\left(2^{\prime}\right)$ holds. Thus $T:=\max \left\{T_{1}, T_{2}\right\}$ is a common squeeze time for $\left\langle N_{1}, N_{0}\right\rangle$ and $\left\langle\tilde{N}_{1}, \tilde{N}_{0}\right\rangle$. The terminology "squeeze time" derives from thinking of the set $N_{1}^{t} \backslash N_{0}^{-t}$ as the result of squeezing the set $N_{1} \backslash N_{0}$ for time $t$ under the action of the flow so that by squeezing for a long enough time it will fit inside a given neighborhood of $S$ (see Figure 1c).

Rather than squeeze $N_{1} \backslash N_{0}$ down to $S$, it is sometimes desirable to squeeze it via the flow down to the local stable or unstable manifold of $S$ within $N_{1}$ where by the local stable (unstable) manifold of $S$ within $N_{1}$ is meant the set of points $x \in N_{1}$ whose positive (negative) semi-orbit lies in $N_{1}$ and whose omega (alpha) limit set lies in $S$ (and in general is not a manifold without additional structure on $S$ ). This is accomplished by considering the families of sets $N_{1}^{t} \backslash N_{0}$ and $N_{1} \backslash N_{0}^{-t}$ for $t \geq 0$; members of the former (latter) family will lie within an arbitrarily small neighborhood relative to $N_{1}$ of the unstable 

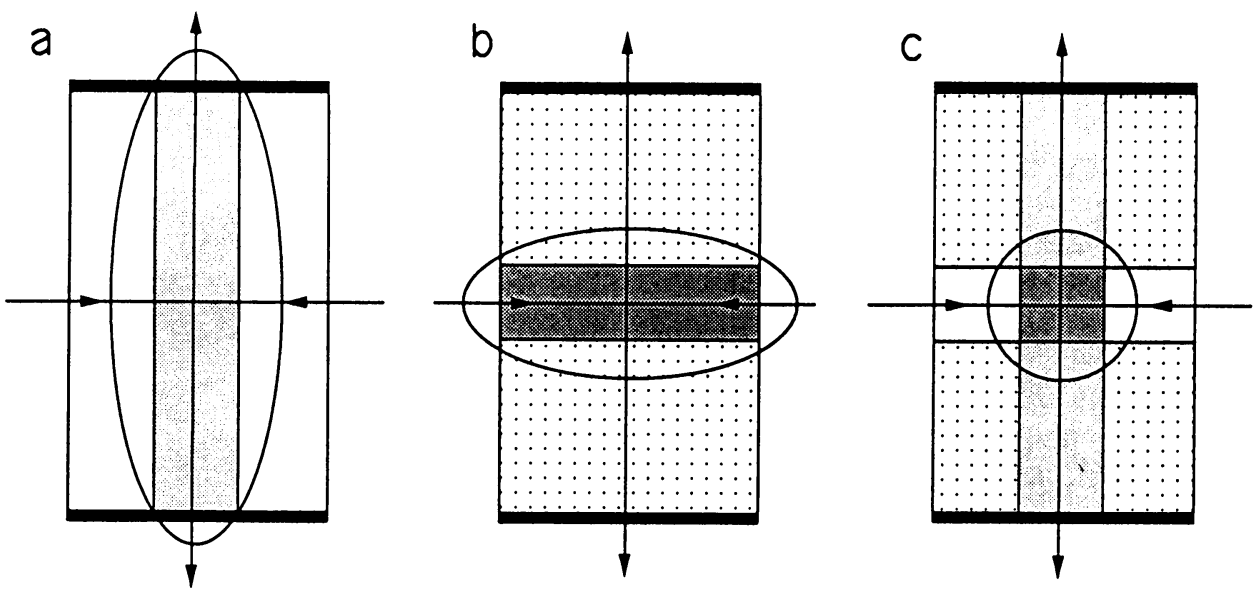

FIGURE 1. Squeezes of an index pair $\left\langle N_{1}, N_{0}\right\rangle$ for the isolated invariant set $S:=\{(0,0)\}$ of the flow on $\mathbf{R}^{2}$ defined by $(x, y) \cdot t:=\left(x e^{-t}, y e^{t}\right)$. In $\mathbf{a}, \mathbf{b}$ and $\mathbf{c}$, the large rectangle is $N_{1}$ and the union of its horizontal edges is $N_{0}$. The ellipses in $\mathbf{a}$ and $\mathbf{b}$, respectively, define neighborhoods, respectively, of the local unstable and stable manifolds of $S$ within $N_{1}$. The circle in c defines a neighborhood of $S$. In a the shaded region is $N_{1}^{t}$; in b the lightly shaded region is $N_{0}^{-t}$ and the heavily shaded region is $N_{1} \backslash N_{0}^{-t}$; in c the darkest region is $N_{1}^{t} \backslash N_{0}^{-t}$.

(stable) manifold of $S$ within $N_{1}$ for large enough $t$ as a consequence of parts (1) and (2) of Lemma 3.3 of [K1] (see Figures 1a and 1b).

As completed in [K1], Conley's notion of morphism between index spaces can be constructed from two types of maps between index spaces, inclusion induced maps and flow induced homeomorphisms, whose definitions follow. Let $\left\langle M_{1}, M_{0}\right\rangle$ and $\left\langle N_{1}, N_{0}\right\rangle$ be index pairs for $S$. Whenever
(i) $M_{1} \backslash M_{0} \subset N_{1}$
and
(ii) $M_{1} \cap M_{0} \cap N_{1} \subset N_{0}$

there is a continuous map $\tilde{i}: M_{1} / M_{0} \rightarrow N_{1} / N_{0}$ induced by these inclusions given by

$$
\tilde{i}\left([x]_{M_{1} / M_{0}}\right):= \begin{cases}{[x]_{N_{1} / N_{0}}} & \text { if } x \in M_{1} \backslash M_{0} \\ {\left[N_{0}\right]} & \text { otherwise }\end{cases}
$$


Also, there is a flow induced homeomorphism $g^{t}: N_{1} / N_{0}^{-t} \rightarrow N_{1}^{t} / N_{0}$ for each $t \geq 0$ defined by

$$
g^{t}([x]):= \begin{cases}{[x \cdot t]} & \text { if } x \in N_{1} \backslash N_{0}^{-t}, \\ {\left[N_{0}\right]} & \text { otherwise. }\end{cases}
$$

Composition of $g^{t}$ with appropriate inclusion induced maps yields maps that are the end maps of a deformation of the index space $N_{1} / N_{0}$. Specifically, for $t \geq 0$, there are clearly inclusion induced maps $\rho^{t}: N_{1} / N_{0} \rightarrow N_{1} / N_{0}^{-t}$ and ${ }^{t}: N_{1}^{t} / N_{0} \rightarrow N_{1} / N_{0}$, and the composites $f_{1}^{t}:=t^{t} \circ g^{t} \circ \rho^{t}: N_{1} / N_{0} \rightarrow N_{1} / N_{0}$ and $\hat{f}_{1}^{t}:=g^{t} \circ \rho^{t}: N_{1} / N_{0} \rightarrow N_{1}^{t} / N_{0}$ are the end maps of the continuous deformation $f^{t}: N_{1} / N_{0} \times[0,1] \rightarrow N_{1} / N_{0}$ defined by

$$
f^{t}([x], s):=f_{s}^{t}([x]):= \begin{cases}{[x \cdot s t]} & \text { if } x \cdot[0, s t] \cap N_{0}=\varnothing, \\ {\left[N_{0}\right]} & \text { otherwise. }\end{cases}
$$

In fact, $f^{t}$ is a weak deformation retraction of $N_{1} / N_{0}$ into $N_{1}^{t} / N_{0}$ where the latter is regarded as a subset of the former via the embedding $t^{t}$. Thus the map $\hat{f}_{1}^{t}$ is the map $f_{1}^{t}$ regarded as a map into $N_{1}^{t} / N_{0}$.

As defined in [K1], the morphisms of $\mathscr{I}(S)$ consist of the homotopy classes of all well-defined, finite compositions of inclusion induced maps and flow induced homeomorphisms between index spaces for $S$; i.e., each morphism is the homotopy class of a map of the form

$$
\varphi:=j_{n} \circ g^{t_{n}} \circ j_{n-1} \circ \cdots \circ j_{1} \circ g^{t_{1}} \circ j_{0}
$$

where each $j_{i}$ is the composition of finitely many inclusion induced maps between index spaces for $S$ and each $g^{t_{i}}$ is a flow-induced homeomorphism. Because each identity map of an index space qualifies as an inclusion induced map, it is immediate with this definition that $\mathscr{I}(S)$ is a category, but it requires some effort to construct such a composite between two arbitrary index spaces (a fairly simple construction is provided by the composite in (4.1) below), and as given in [K1] the proof that there is exactly one such morphism from one index space to another, specifically, to prove that any such composition from an index space back to itself is homotopic to the identity map on that index space, is fairly involved. The hard part of the proof in [K1] is showing that given $\varphi: N_{1} / N_{0} \rightarrow N_{1} / N_{0}$ as in (3.1), there exists $r>0$ so that where $\rho^{r}: N_{1} / N_{0} \rightarrow N_{1} / N_{0}^{-r}$ is inclusion induced, where $\bar{t}=\sum_{i=1}^{n} t_{i}$, and where $f_{1}^{\bar{t}}$ is the endmap of the deformation $f^{\bar{t}}$ of $N_{1} / N_{0}$, the equality $\rho^{r} \circ \varphi=\rho^{r} \circ f_{1}^{\bar{t}}$ holds. Once this is shown and with the equivalence relation of maps being basepoint homotopic denoted by " $\sim$ ", the string of relations

$$
\begin{aligned}
\varphi & =1_{N_{1} / N_{0}} \circ \varphi \sim f_{1}^{r} \circ \varphi \\
& =\left(l^{r} \circ g^{r} \circ \rho^{r}\right) \circ \varphi=\left(l^{r} \circ g^{r}\right)\left(\rho^{r} \circ \varphi\right) \\
& =\left(l^{r} \circ g^{r}\right)\left(\rho^{r} \circ f_{1}^{\bar{t}}\right)=\left(l^{r} \circ g^{r} \circ \rho^{r}\right) \circ f_{1}^{\bar{t}} \\
& =f_{1}^{r} \circ f_{1}^{\bar{t}} \sim 1_{N_{1} / N_{0}} \circ 1_{N_{1} / N_{0}}=1_{N_{1} / N_{0}}
\end{aligned}
$$


shows that $\varphi$ is homotopic to the identity, and as with the previous definition it follows that each map used to define a morphism in the Conley index has a map of the same type for homotopy inverse, hence is a homotopy equivalence. Uniqueness of inverses yields that any two composites of inclusion induced maps and flow induced homeomorphisms with common domain and range index spaces are homotopy equivalent.

The maps $\varphi$ and $f_{1}^{\bar{t}}$ above would in fact be equal and all of the difficulties in the proof just outlined would disappear were it not for the fact that the notion of inclusion induced map between index spaces defined above is not functorial; i.e., the composition of two inclusion induced maps need not yield a map that is inclusion induced. For example, take the flow on $\mathbf{R}$ defined by $x \cdot t=x e^{t}$ which has $S:=\{0\}$ as its only nonempty isolated invariant set and choose six real numbers $a_{i}, b_{i}, i=1,2,3$, satisfying $0<b_{1}<b_{2}<a_{1}<b_{3}<a_{2}<a_{3}$. Then with $N_{1 i}:=\left[-a_{i}, a_{i}\right]$ and $N_{0 i}:=\left[-a_{i},-b_{i}\right] \cup\left[b_{i}, a_{i}\right]$, the pair $\left\langle N_{1 i}, N_{0 i}\right\rangle$ is an index pair for $S, i=1,2,3$, and for $j=1,2$ there is an inclusion induced map $l_{j+1 j}: N_{1 j+1} / N_{0 j+1} \rightarrow N_{1 j} / N_{0 j}$. However, the composite $l_{21} \circ l_{32}$ is not an inclusion induced map from $N_{13} / N_{03}$ to $N_{11} / N_{01}$ since $N_{13} \backslash N_{03}=$ ]$-b_{3}, b_{3}\left[\not \subset\left[-a_{1}, a_{1}\right]\right.$.

In showing that the two definitions of the Conley index are equivalent, it is clearly desirable to avoid having to appeal to the difficult proof of [K1]. The following three propositions allow this circumvention.

Let $\left\langle N_{1}, N_{0}\right\rangle$ and $\left\langle\tilde{N}_{1}, \tilde{N}_{0}\right\rangle$ be index pairs for $S$ and $T \geq 0$ a common squeeze time for these pairs.

3.1 Proposition. If there is an inclusion induced map $\tilde{i}: N_{1} / N_{0} \rightarrow \tilde{N}_{1} / \tilde{N}_{0}$, then it is homotopic to each flow map $F^{t}: N_{1} / N_{0} \rightarrow \tilde{N}_{1} / \tilde{N}_{0}$ for $t \geq T$.

Proof. Let us use $\tilde{F}^{t}: \tilde{N}_{1} / \tilde{N}_{0} \rightarrow N_{1} / N_{0}$ to denote the flow map for $t \geq T$. It will suffice to show that each composite $\tilde{F}^{t} \circ \tilde{l}$ is homotopic to the identity map $1_{N_{1} / N_{0}}$. For if this is so and if the equivalence relation of maps being basepoint homotopic is denoted by " $\sim$ ", then the string of relations

$$
\tilde{l}=1_{\tilde{N}_{1} / \tilde{N}_{0}} \circ \tilde{l} \sim\left(F^{t} \circ \tilde{F}^{t}\right) \circ \tilde{\imath} \sim F^{t} \circ\left(\tilde{F}^{t} \circ \tilde{l}\right) \sim F^{t} \circ 1_{N_{1} / N_{0}}=F^{t}
$$

shows that $\tilde{l}$ is homotopic to a flow map. The index pair $\left\langle N_{1}, N_{0}\right\rangle$ taken twice, as already observed, has common squeeze time zero, and let us use $F_{0}^{t}: N_{1} / N_{0} \rightarrow$ $N_{1} / N_{0}$ to denote the flow map for $t \geq 0$. Observe that the composite $\tilde{F}^{t} \circ \tilde{l}$ is given by the formula

$$
\tilde{F}^{t} \circ \tilde{l}([x])= \begin{cases}{[x \cdot 3 t]} & \text { if (a) } x \in N_{1} \backslash N_{0} \cap \tilde{N}_{1} \backslash \tilde{N}_{0} \\ & \text { and (b) } x \cdot[0,2 t] \subset \tilde{N}_{1} \backslash \tilde{N}_{0} \\ & \text { and (c) } x \cdot[t, 3 t] \subset N_{1} \backslash N_{0} \\ & \text { otherwise. }\end{cases}
$$

Clearly, if $x$ satisfying conditions (a), (b), and (c) is equivalent to $x$ satisfying (d) $x \cdot[0,3 t] \subset N_{1} \backslash N_{0}$, then $\tilde{F}^{t} \circ \tilde{l}=F_{0}^{t}$ when $t \geq T$. 
To show the equivalence, observe that by condition (ii) in the definition of an inclusion induced map, the relation

$$
N_{1} \cap N_{0} \cap \tilde{N}_{1} \backslash \tilde{N}_{0}=\varnothing
$$

holds. Thus if $s>0$ and $x \cdot\left[0, s\left[\subset N_{1} \backslash N_{0}\right.\right.$, but $x \cdot s \in N_{0}$, it follows that $x \cdot[0, s] \not \subset \tilde{N}_{1} \backslash \tilde{N}_{0}$. It then follows from the exit property of the index pair $\left\langle N_{1}, N_{0}\right\rangle$ that for $t \geq 0$, if

(a') $x \in N_{1} \backslash N_{0}$ and

(b') $x \cdot[0, t] \subset \tilde{N}_{1} \backslash \tilde{N}_{0}$,

then $x \cdot[0, t] \subset N_{1} \backslash N_{0}$. Since conditions $\left(\mathrm{a}^{\prime}\right)$ and $\left(\mathrm{b}^{\prime}\right)$ are immediate from (a) and (b), respectively, it follows that (a), (b), and (c) together imply (d).

Conversely, when $x$ satisfies (d) and $t \geq T$, it certainly satisfies (c), is an element of $N_{1} \backslash N_{0}$, and by defintion of $T, x \cdot[t, 2 t] \subset \tilde{N}_{1} \backslash \tilde{N}_{0}$. In fact $x \cdot[0,2 t] \subset \tilde{N}_{1} \backslash \tilde{N}_{0}$; whence, (a) and (b) are satisfied. For when $x$ satisfies (d), since $N_{1} \backslash N_{0} \subset \tilde{N}_{1}$ by condition (i) in the definition of an inclusion induced map, $x \cdot[0, t] \subset \tilde{N}_{1}$, and in fact $x \cdot[0, t] \subset \tilde{N}_{1} \backslash \tilde{N}_{0}$; otherwise the positive invariance of $\tilde{N}_{0}$ relative to $\tilde{N}_{1}$ would imply that $x \cdot t \in \tilde{N}_{0}$ in contradiction to the already observed fact that (d) implies $x \cdot t \notin \tilde{N}_{0}$ when $t \geq T$.

Thus $\tilde{F}^{t} \circ \tilde{l}=F_{0}^{t}$ when $t \geq T$, but $F_{0}^{t}$, is homotopic to $F_{0}^{0} \equiv 1_{N_{1} / N_{0}}$ for each $t \geq 0$. Thus $\tilde{F}^{t} \circ \tilde{l} \sim 1_{N_{1} / N_{0}}$ as desired.

3.2 Proposition. The index pairs $\left\langle N_{1}, N_{0}^{-t}\right\rangle$ and $\left\langle N_{1}^{t}, N_{0}\right\rangle$ have.common squeeze time $t$, and the flow induced homeomorphism $g^{t}: N_{1} / N_{0}^{-t} \rightarrow N_{1}^{t} / N_{0}$ is homotopic to the flow map $F^{t}: N_{1} / N_{0}^{-t} \rightarrow N_{1}^{t} / N_{0}$.

Proof. Define $G: N_{1} / N_{0}^{-t} \times\left[t, \infty\left[\rightarrow N_{1}^{t} / N_{0}\right.\right.$ by

$$
G([x], s):=G^{s}([x]):= \begin{cases}{[x \cdot s]} & \text { if } x \cdot[0, s] \subset N_{1} \backslash N_{0}, \\ {\left[N_{0}\right]} & \text { otherwise. }\end{cases}
$$

The proof that $G$ is continuous is similar to the proof in [S] that flow maps are continuous and is left to the reader. Also, observe that the condition $x \cdot[0,3 t] \subset$ $N_{1} \backslash N_{0}$ is equivalent to the compound condition $x \cdot[0,2 t] \subset N_{1} \backslash N_{0}^{-t}$ and $x \cdot[t, 3 t] \subset N_{1}^{t} \backslash N_{0}$. Since it follows straightforwardly from the definitions that $t$ is a common squeeze time for the index pairs $\left\langle N_{1}, N_{0}^{-t}\right\rangle$ and $\left\langle N_{1}^{t}, N_{0}\right\rangle$, it follows immediately that $G^{(1-r) t+r(3 t)}, 0 \leq r \leq 1$, is a homotopy from $g^{t}$ to the flow map $F^{t}$.

3.3 Proposition. Every map $\varphi$ which is a composite of inclusion induced maps and flow induced homeomorphisms between index spaces for $S$ is homotopic to a flow map. In particular, the endmaps $f_{1}^{t}$ and $\hat{f}_{1}^{t}$ of the deformation $f^{t}: N_{1} / N_{0} \times$ $[0,1] \rightarrow N_{1} / N_{0}$ are homotopic to flow maps.

Proof. The first statement is an immediate consequence of the previous two propositions and the functoriality of flow maps; i.e., that the composition of 
the homotopy classes of two flow maps is the homotopy class of a flow map. The second follows from the first and the factorizations of $f_{1}^{t}$ and $\hat{f}_{1}^{t}$ given above.

\section{EQUIVALENCE OF THE DEFINITIONS}

Let $\left\langle N_{1}, N_{0}\right\rangle$ and $\left\langle\tilde{N}_{1}, \tilde{N}_{0}\right\rangle$ be index pairs for $S$ and let $T$ be a common squeeze time for these index pairs. That the two definitions of the Conley index given above yield the same small category is an immediate consequence of the following result.

4.1 Theorem. For $t \geq T$, the flow map $F^{t}: N_{1} / N_{0} \rightarrow \tilde{N}_{1} / \tilde{N}_{0}$ has the factorization

$$
N_{1} / N_{0} \rightarrow N_{1} / N_{0}^{-t} \stackrel{\hat{f}_{1}^{t}}{\longrightarrow} N_{1}^{t} / N_{0}^{-t} \rightarrow \tilde{N}_{1} / \tilde{N}_{0}^{-2 t} \stackrel{g^{2 t}}{\longrightarrow} \tilde{N}_{1}^{2 t} / \tilde{N}_{0} \rightarrow \tilde{N}_{1} / \tilde{N}_{0}
$$

where each of the unlabled arrows is an inclusion induced map and $\hat{f}_{1}^{t}$ is the endmap of the deformation $f^{t}$ of the index space $N_{1} / N_{0}^{-t}$. Hence, each flow map is a composition of inclusion induced maps and flow induced homeomorphisms between index spaces for $S$. Conversely, any composite of inclusion induced maps and flow induced homeomorphisms between index spaces for $S$ is homotopic to a flow map.

Proof. For the composite in (4.1) to be well-defined it suffices to show that the inclusion induced map $N_{1}^{t} / N_{0}^{-t} \rightarrow \tilde{N}_{1} / \tilde{N}_{0}^{-2 t}$ is well-defined since the other factors clearly are. Let us assume for the moment the well-definedness of this factor whenever $t \geq T$. It is a simple observation that if the conditions (i) $x$. $[0,2 t] \subset N_{1} \backslash N_{0}$ and (ii) $x \cdot[t, 3 t] \subset \tilde{N}_{1} \backslash \tilde{N}_{0}$ are satisfied then the composite maps $[x]$ to $[x \cdot 3 t] \neq\left[\tilde{N}_{0}\right]$. On the other hand, if condition (i) fails then $[x]$ maps to the base point by the time of its passage through the second factor, and if condition (ii) fails then $[x]$ maps to the base point no later than the third factor. Thus once the composite is shown well-defined it is indeed a factorization of $F^{t}: N_{1} / N_{0} \rightarrow \tilde{N}_{1} / \tilde{N}_{0}$. The converse is Proposition 3.3 above.

It remains to show that there is an inclusion induced map $N_{1}^{t} / N_{0}^{-t} \rightarrow \tilde{N}_{1} / \tilde{N}_{0}^{-2 t}$ whenever $t \geq T$. It is immediate that $N_{1}^{t} \backslash N_{0}^{-t} \subset \tilde{N}_{1} \backslash \tilde{N}_{0}$ by property $\left(1^{\prime}\right)$; whence the first condition for having an inclusion induced map follows. Verifying the second condition is harder. It must be shown that

$$
N_{1}^{t} \cap N_{0}^{-t} \cap \tilde{N}_{1} \subset \tilde{N}_{0}^{-2 t}
$$

Suppose not; i.e., suppose there exists

$$
x \in N_{1}^{t} \cap N_{0}^{-t} \cap \tilde{N}_{1} \backslash \tilde{N}_{0}^{-2 t} .
$$

Set $\tilde{M}:=\operatorname{cl}\left(\tilde{N}_{1} \backslash \tilde{N}_{0}\right)$, and note that $\tilde{M}$ isolates $S$. It is straightforward to verify that

$$
\left\langle N_{1}^{t} \cap \tilde{M}, N_{1}^{t} \cap N_{0}^{-t} \cap \tilde{M}\right\rangle \quad \text { and } \quad\left\langle\tilde{N}_{1} \cap \tilde{M}, \tilde{N}_{0} \cap \tilde{M}\right\rangle
$$


are index pairs for $S$ relative to the isolating neighborhood $\tilde{M}$; i.e., for both pairs, each member of the pair is a subset of $\tilde{M}$, and if $K$ is either member of either pair, $x \in K$ and $x \cdot[0, s] \subset \tilde{M}$, some $s \geq 0$, then $x \cdot[0, s] \subset K$. For details see the proof of [K1, Proposition 3.4].

Because $x \in \tilde{N}_{1} \backslash \tilde{N}_{0}^{-2 t}$, it follows that

$$
x \cdot t \cdot[-t, t] \subset \tilde{N}_{1} \backslash \tilde{N}_{0}
$$

hence,

$$
x \cdot t \in N_{1} \backslash N_{0} .
$$

Now either $x \cdot[0, t] \subset \tilde{M}$ or not. If the inclusion holds, then since $x \in$ $N_{1}^{t} \cap N_{0}^{-t} \cap \tilde{M}$, the positive invariance of an index pair relative to $\tilde{M}$ implies $x \cdot t \in N_{1} \cap N_{0}$, contradicting (4.3). On the other hand, if the inclusion fails, then since $x \in \tilde{N}_{1} \cap \tilde{M}$, the exit property implies that

$$
x \in\left(\tilde{N}_{0} \cap \tilde{M}\right)^{-t} \subset \tilde{N}_{0}^{-2 t},
$$

contradicting (4.2).

Remark. The factorization of $F^{t}$ into the composite of inclusion induced maps and flow induced homeomorphisms in (4.1) gives a much simpler construction of such a map between a pair of arbitrary index spaces than the composite $m$ defined in the proof of [K1, Proposition 3.4], but is similar in that an examination of that proof shows that

$$
m([x]):= \begin{cases}{\left[x \cdot\left(r+r^{\prime}+s\right)\right]} & \text { if } x \cdot\left[0,2\left(r+r^{\prime}\right)\right] \subset N_{1} \backslash N_{0} \\ & \text { and } x \cdot\left[r+r^{\prime}, r+r^{\prime}+s\right] \subset \tilde{N}_{1} \backslash \tilde{N}_{0}, \\ {\left[\tilde{N}_{0}\right]} & \text { otherwise }\end{cases}
$$

where $r \geq 0$ and $s \geq r^{\prime} \geq 0$ are chosen so that property $\left(1^{\prime}\right)$ holds when $t:=r$ (hence also for $\left.t:=r+r^{\prime}\right)$ and $\left(N_{1}^{r} \cap \tilde{N}_{1}\right)^{r^{\prime}} \cap\left(N_{0}^{-r} \cap N_{1} \cap \tilde{N}_{1}\right)^{-r^{\prime}} \cap \tilde{N}_{1} \subset \tilde{N}_{0}^{-s}$. Thus $m$ coincides with the flow map $F^{r+r^{\prime}}$ for $s:=2\left(r+r^{\prime}\right)$ and $r$ taken sufficiently large.

The next proposition illustrates one situation in which it is important to know that an inclusion induced map between index pairs for $S$ has homotopy class that is a morphism in $\mathscr{I}(S)$ and at the same time points out a situation where one wants to squeeze $N_{1} \backslash N_{0}$ to the stable manifold of $S$ within $N_{1}$. Its use as a lemma in the main theorem of [K5] will be briefly discussed following the proof. The proof is based on Figure $2 \mathrm{a}$ which is arrived at by considering the flow on the $x-y$ plane given by $(x, y) \cdot t:=\left(x e^{-t}, y e^{t}\right)$. The origin is a saddle and is isolated by any rectangular 2 -cell with its edges parallel to the coordinate axes and containing the origin in its interior. Let $N_{1}$ be one such 2-cell, and let $N_{0}$ be the union of its edges parallel to the $x$-axis. Then $\left\langle N_{1}, N_{0}\right\rangle$ is an index pair for $\{(0,0)\}$, and $N_{1} / N_{0}$ has the homotopy type of a circle with basepoint. Hence, $\widetilde{H}_{1}\left(N_{1} / N_{0}\right)$, the one-dimensional reduced singular homology group of 

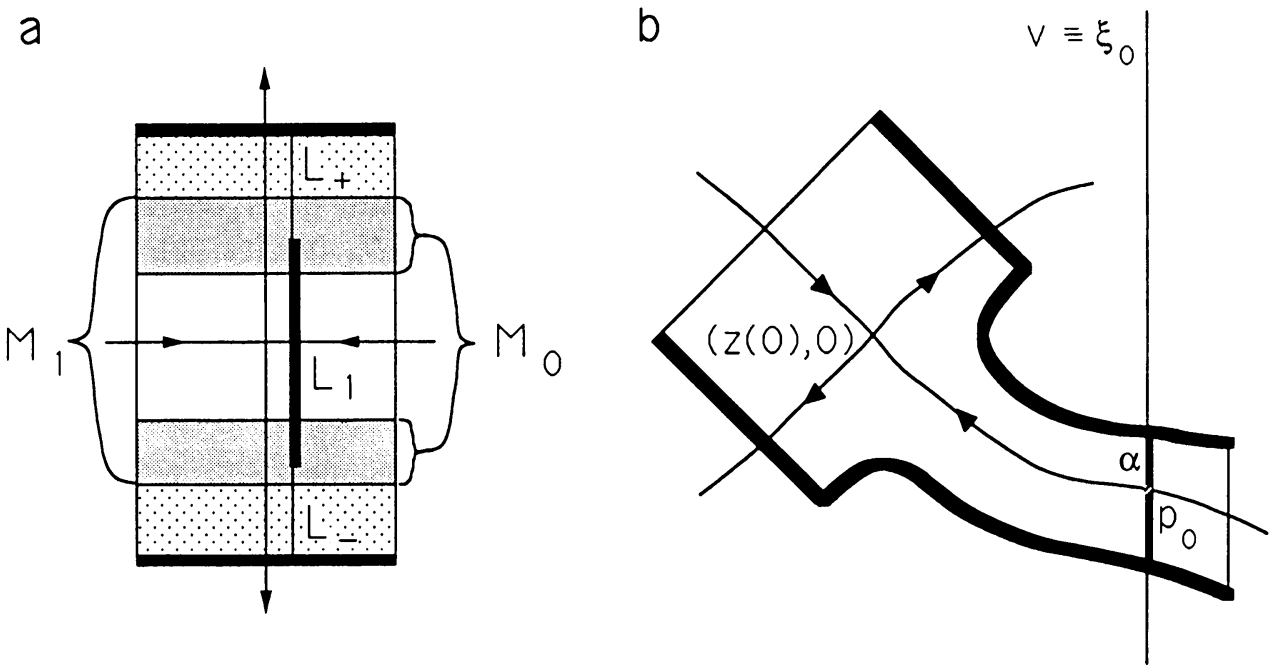

FIGURE 2. In a the largest rectangle is $N_{1}$, the lightly shaded region is $N_{1}^{-s}$ and the union of the shaded regions is $N_{1}^{-t}$. The edges of the topological 2-cell in b are transverse to the flow of the planar system $(v, w)^{\circ}=$ $(w, f(v, 0))$ where $f(z(0), 0)=0$ and $f_{v}(z(0), 0)>$ 0 . The 2-cell together with its heavily drawn edges forms an index pair for the saddle point $(z(0), 0)$ that is homeomorphic to the index pair $\left\langle N_{1}, N_{0}\right\rangle$ in a via a homeomorphism that sends $\alpha$ onto $L=L_{-} \cup L_{1} \cup L_{+}$.

$N_{1} / N_{0}$, is isomorphic to $\mathbf{Z}$. Thus $\widetilde{H}_{1}\left(M_{1} / M_{0}\right)$ is isomorphic to $\mathbf{Z}$ for each index pair $\left\langle M_{1}, M_{0}\right\rangle$ for $\{(0,0)\}$.

In the general case for $S$ an arbitrary isolated invariant set, define an equivalence relation on homology classes $\nu \in \widetilde{H}_{*}\left(M_{1} / M_{0}\right)$ as $\left\langle M_{1}, M_{0}\right\rangle$ ranges over all index pairs for $S$ by $\nu \in \widetilde{H}_{*}\left(M_{1} / M_{0}\right)$ is equivalent to $\nu^{\prime} \in \widetilde{H}_{*}\left(M_{1}^{\prime} / M_{0}^{\prime}\right)$ if, and only if, $h_{*} \boldsymbol{\nu}=\boldsymbol{\nu}^{\prime}$ where $h_{*}$ is the map on homology induced by the unique homotopy class $h: M_{1} / M_{0} \rightarrow M_{1}^{\prime} / M_{0}^{\prime}$ which is a morphism in $\mathscr{I}(S)$. Each equivalence class under this relation has a unique representative in the homology group of any index space for $S$; whence the addition in any such homology group defines an addition in the set of equivalence classes, which is easily seen to be independent of the particular index space used to define it. Thus the set of equivalence classes is a group, called the homology of the Conley index of $S$ and denoted $\widetilde{H}_{*} \mathscr{I}(S)$. In Figure 2a, any vertical line segment in 
$N_{1}$ with its endpoints in $N_{0}$ is a geometric representative of a generator of that group. Specifically, let $\sigma$ be a homeomorphism of $[0,1]$ onto one such line segment $L$. Then $\sigma$ is a singular 1-cycle in $N_{1}$ modulo $N_{0}$ whose homology class generates $H_{1}\left(N_{1}, N_{0}\right)$ and whose image under the quotient map $N_{1} \rightarrow N_{1} / N_{0}$ generates $\widetilde{H}_{1}\left(N_{1} / N_{0}\right)$ and thereby represents a generator of $\widetilde{H}_{*} \mathscr{I}(S)$, call it $\boldsymbol{\alpha}$. In general, given an index pair $\left\langle N_{1}, N_{0}\right\rangle$ with $N_{1} \supset N_{0}$ (such a pair is called a nested index pair), a singular $n$-chain $\alpha$ in $N_{1}$ is an $n$-cycle in $N_{1}$ modulo $N_{0}$ if $\partial \alpha$ is a chain in $N_{0}$ and has homology class $\langle\alpha\rangle \in H_{n}\left(N_{1}, N_{0}\right)$ representing the homology class $\alpha \in \widetilde{H}_{n} \mathscr{I}(S)$ where $\alpha$ is the equivalence class of $q_{*}\langle\alpha\rangle$ and $q:\left(N_{1}, N_{0}\right) \rightarrow\left(N_{1} / N_{0},\left[N_{0}\right]\right)$ is the quotient map.

In the next proposition, by a subchain of a singular chain $\alpha$ is meant a chain obtained by deleting some of the summands in any iterated barycentric subdivision of $\alpha$. Also, let $A^{+}\left(N_{1}, S\right)$ denote the local stable manifold of $S$ within $N_{1}$.

4.2 Proposition. Let $\left\langle N_{1}, N_{0}\right\rangle$ be a nested index pair for $S$ and let $\alpha$ be a singular cycle in $N_{1}$ modulo $N_{0}$ representing nonzero homology $\boldsymbol{\alpha} \in \widetilde{H}_{*} \mathscr{I}(S)$. Then (1) there exists a sequence of nested index pairs $\left\langle M_{1 n}, M_{0 n}\right\rangle, n \in \mathbf{Z}^{+}$, starting at $\left\langle N_{1}, N_{0}\right\rangle$ and satisfying $M_{1 n} \subset$ int $_{N_{1}}\left(M_{1 n-1}\right)$ and $\bigcap_{n=0}^{\infty} M_{1 n}=A^{+}\left(N_{1}, S\right)$, and (2) there exists a sequence $\left\{\alpha_{n}\right\}_{n=0}^{\infty}$ of singular chains starting at $\alpha$ and with $\alpha_{n+1}$ a subchain of $\alpha_{n}, n \geq 0$, and $\alpha_{n}$ a cycle in $M_{1 n}$ modulo $M_{0 n}$ representing $\boldsymbol{\alpha}$.

Proof. Choose $t>s>0$ and define

$$
\left\langle M_{1}, M_{0}\right\rangle:=\left\langle\mathrm{cl}\left(N_{1} \backslash N_{0}^{-s}\right), N_{0}^{-t} \cap \mathrm{cl}\left(N_{1} \backslash N_{0}^{-s}\right)\right\rangle .
$$

Then $\left\langle M_{1}, M_{0}\right\rangle$ is an index pair for $S$, and if $t$ and $s$ are chosen large enough, $M_{1}$ is disjoint from $N_{0}$ and

$$
G:=\left\{N_{1} \backslash N_{0}^{-s}, \text { int }_{N_{1}}\left(N_{0}^{-t} \cap N_{1}\right)\right\}
$$

is an $N_{1}$-open cover of $N_{1}$ because given $s^{\prime} \geq 0$, it is possible [K1, Proposition 2.9] to choose $s^{\prime \prime}>s^{\prime}$ so that $N_{0}^{-s^{\prime}} \cap N_{1} \subset \operatorname{int}_{N_{1}}\left(N_{0}^{-s^{\prime \prime}} \cap N_{1}\right)$. Application of some sufficiently high iterate of the barycentric subdivison operator, say the $k^{\text {th }}$, to $\alpha$ yields a chain subordinate to the cover $G$; i.e., $\operatorname{sd}^{k} \alpha=\alpha_{1}+\alpha^{\prime}$ where $\alpha_{1}$ is a chain in $N_{1} \backslash N_{0}^{-s}$, hence in $M_{1}$, and $\alpha^{\prime}$ is a chain in $\operatorname{int}_{N_{1}}\left(N_{0}^{-t} \cap N_{1}\right)$, hence in $N_{0}^{-t} \cap N_{1}$. In Figure 2a this corresponds to division of $L$ into three segments so that the middle segment $L_{1}$ lies in the interior of $M_{1}$ relative to $N_{1}$ with one endpoint in each component of $M_{0}$ and so that the outer two segments $L_{-}, L_{+}$lie interior to $N_{0}^{-t} \cap N_{1}$ relative to $N_{1}$. Thus $L_{1}$ is the support of a 1-cycle in $M_{1}$ modulo $M_{0}$. In general let us show that $\alpha_{1}$ is a cycle in $M_{1}$ modulo $M_{0}$ and represents $\alpha \in \widetilde{H}_{*} \mathscr{I}(S)$.

Because the subdivision operator is constructed as a natural chain map the boundary chains $\partial\left(\alpha_{1}+\alpha^{\prime}\right)$ and $\partial \alpha$ have the same support in $N_{0}$, but $\alpha_{1}$ is 
a chain in $M_{1}$ which is disjoint from $N_{0}$. Thus each summand of $\partial \alpha_{1}$ must cancel with a summand in $\partial \alpha^{\prime}$, and since $\partial \alpha^{\prime}$ is a chain in $N_{0}^{-t} \cap N_{1}, \partial \alpha_{1}$ is a chain in $M_{1} \cap N_{0}^{-t}=M_{0}$. Thus, $\alpha_{1}$ is a cycle in $M_{1}$ modulo $M_{0}$ and by construction is a subchain of $\alpha$. To see that $\alpha_{1}$ represents $\alpha \in \widetilde{H}_{*} \mathcal{I}(S)$, note that the commutativity of the left-hand rectangle in the diagram

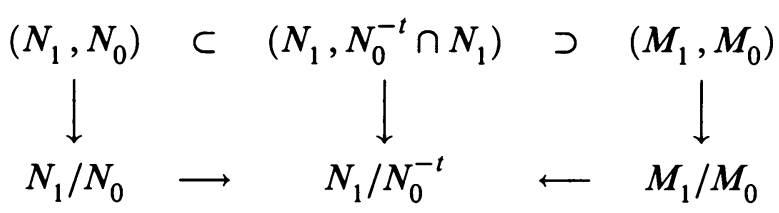

where the vertical arrows are the quotient maps and the bottom horizontal arrows are the inclusion induced maps implies that $\alpha$ as a cycle in $N_{1}$ modulo $N_{0}^{-t} \cap N_{1}$ represents $\boldsymbol{\alpha}$ because the homotopy class of the inclusion induced map is a morphism in $\mathscr{I}(S)$. Thus as $\operatorname{sd}^{k} \alpha$ is chain homotopic to $\alpha$, also $\alpha_{1}+\alpha^{\prime}$ as a cycle in $N_{1}$ modulo $N_{0}^{-t} \cap N_{1}$ represents $\boldsymbol{\alpha}$. Then as $\alpha^{\prime}$ is a chain in $N_{0}^{-t} \cap N_{1}$, by definition of two relative cycles being homologous, $\alpha_{1}$ as a cycle in $N_{1}$ modulo $N_{0}^{-t} \cap N_{1}$ represents $\boldsymbol{\alpha}$. Finally, the commutativity of the righthand rectangle in the above diagram shows that, as a cycle in $M_{1}$ modulo $M_{0}$, $\alpha_{1}$ represents $\boldsymbol{\alpha}$, again because the homotopy class of the inclusion induced map is a morphism in $\mathscr{S}(S)$.

Thus the sequence of index pairs starts with $M_{j 0}:=N_{j}$ and $M_{j 1}:=M_{j}$, $j=0,1$, and the sequence of chains starts with $\alpha_{0}:=\alpha$ and $\alpha_{1}$ as chosen above; also set $s_{1}:=s$. Repeating the above construction with the index pair $\left\langle M_{1}, M_{0}\right\rangle$ taking the role of $\left\langle N_{1}, N_{0}\right\rangle$ and $\alpha_{1}$ the role of $\alpha$ and then iterating ad infinitum produces the desired sequence of index pairs and chains with the proviso that $s_{n} \uparrow \infty$ where $t_{n}>s_{n}, n \geq 1$, are the times used to construct $\left\langle M_{1 n}, M_{0 n}\right\rangle$ from $\left\langle M_{1 n-1}, M_{0 n-1}\right\rangle$ to ensure that $\bigcap M_{1 n}=A^{+}\left(N_{1}, S\right)$.

The intent of the following rather sketchy description is to give the reader a brief indication of how the previous proposition is used to establish the presence of layers in solutions of a class of two-point boundary value problems. In [K5] existence theorems for vector two-point problems of the form $\varepsilon \mathbf{u}^{\prime}=\mathbf{F}(\mathbf{u}, x, \varepsilon)$, $\mathbf{u}(\lambda) \in \mathbf{E}(\lambda), \lambda=0,1$, are established which describe the presence of interior transition layers and endpoint layers, as appropriate, and are based on the existence of curves of sets $x \mapsto S(x)$ where $S(x)$ is an isolated invariant set of the associated fast system at $x, \dot{\mathbf{u}}=\mathbf{F}(\mathbf{u}, x, 0)$ where a dot denotes differentiation with respect to $t$ and $\dot{x}=0$. The proofs are based on a generalization of Wazewski's principle deriving from the continuation properties of the Conley index, and generalize results proved in [B] for the scalar problem $\varepsilon^{2} v^{\prime \prime}=f(v, x)$, $v(\lambda)=\xi_{\lambda}, \lambda=0,1$, by applying Nagumo's method of strict lower and upper solutions. Strict lower and upper solutions, $v=\underline{z}_{\varepsilon}(x)$ and $v=\bar{z}_{\varepsilon}(x)$, are constructed from a curve (or curves) $v=z(x)$ defining an isolated branch of solutions of $f(v, x)=0$ on which the partial derivative $f_{v}(z(x), x)$ is positive. 
Note that the scalar problem takes the form of the vector problem if we define $\mathbf{u}:=(v, w), \mathbf{F}(\mathbf{u}, x):=(w, f(v, x))$, and $\mathbf{E}(\lambda):=\left\{(v, w): v=\xi_{\lambda}\right\}$. As part of the hypotheses in [K5] it is assumed that the endpoint set $\mathbf{E}(0)$ contains the support of a singular chain $\alpha$ representing nonzero homology in $\widetilde{H}_{*} \mathscr{I}(S(0))$ as motivated by the following discussion of the scalar problem.

The assumption on the positivity of $f_{v}$ implies via linearization that $(z(x), 0)$ is a saddle point of the associated fast planar system $(v, w)^{\circ}=(w, f(v, x))$. Thus with $S(x):=\{(z(x), 0)\}$ we get a curve of isolated invariant sets for the fast systems. The typical manner in which the line $v=\xi_{0}$ contains the support of a 1-chain $\alpha$ representing nonzero homology $\alpha \in \widetilde{H}_{1} \mathscr{I}((z(x), 0))$ is depicted in Figure $2 \mathbf{b}$. Then $\alpha$, regarded as a subset of $\mathbf{R}^{2} \times\{0\}$, when followed under the flow on $\mathbf{R}^{3}$ of the system $\varepsilon \mathbf{u}^{\prime}=\mathbf{F}(\mathbf{u}, x), x^{\prime}=1,0<\varepsilon \ll 1$, has image in $\mathbf{R}^{2} \times\{1\}$ which is forced by additional hypotheses too lengthy to describe here to intersect the line $v=\xi_{1}$ regarded as a subset of $\mathbf{R}^{2} \times\{1\}$ thereby determining a solution of the problem. The previous proposition allows the argument to be applied to a sequence of subchains of $\alpha$ collapsing to $p_{0}$ with $\varepsilon$ forced to decrease as the subchain decreases. The family of solutions so found exhibits a layer at $x=0$ since the initial points of these solutions limit on $p_{0}$ and since in the stretched time parameterizations $(t=x / \varepsilon)$, there is an initial segment of each solution that closely approximates an initial segment of the positive semi-orbit through $p_{0}$ with respect to the fast flow at $x=0$ with the initial segments converging to the full semi-orbit as $\varepsilon$ decreases to zero; i.e., as $\varepsilon$ decreases to zero there are initial segments of the solution curves that converge to the segment of the stable manifold of $(z(0), 0)$ bounded by it and $p_{0}$.

\section{REFERENCES}

[B] Yu. P. Boglaev, The two-point problem for a class of ordinary differential equations with a small parameter coefficient of the derivative, USSR Comput. Math.-Math. Phys. 10 (1970), 190-204.

[C] C.C. Conley, Isolated Invariant Sets and the Morse Index, CBMS Conference Proceedings, Amer. Math. Soc., Providence, R.I., 1978.

[K1] H.L. Kurland, The Morse index of an isolated invariant set is a connected simple system, J. Differential Equations 42 (1981), 234-259.

[K2] _ Homotopy invariants of a repeller-attractor pair, I: the Püppe sequence of an R-A pair, J. Differential Equations 46 (1982), 1-31.

[K3] _ Homotopy invariants of a repeller-attractor pair, II: continuation of an $R$-A pair, J. Differential Equations 49 (1983), 281-329.

[K4] _ Following homology in singularly perturbed systems, J. Differential Equations 62 (1986), 1-72.

[K5] L L Layers in singularly perturbed systems via homology contintuation (to appear).

[S] D. Salamon, Connected simple systems and the Conley index of isolated invariant sets, Trans. Amer. Math. Soc. 291 (1985), 1-41.

9 Mohawk Trail, Clifton Park, New York 12065 